\title{
UTILIZANDO A ANÁLISE DE CITAÇÕES DE TESES PARA AVALIAR A COLEÇÃO DE LIVROS EM BIBLIOTECAS UNIVERSITÁRIAS
} USING CITATION ANALYSIS FOR EVALUATE BOOKS COLLECTION IN AN ACADEMIC LIBRARY

\author{
Ana Maria Mattos \\ Universidade Federal do Rio Grande do Sul \\ ammattos@ea.ufrgs.br \\ Tania Marisa de Abreu Fraga \\ Universidade Federal do Rio Grande do Sul \\ tmafrga@ea.ufrgs.br
}

\begin{abstract}
Resumo
O objetivo desta pesquisa é determinar a adequação ou não da coleção de livros das bibliotecas da Universidade Federal do Rio Grande do Sul (UFRGS) para os alunos do curso de doutorado em Administração. A metodologia verifica a correlação existente entre os livros citados nas teses de 1999 até 2007 e o acervo disponível nas bibliotecas. A investigação conclui que a coleção de livros nacionais apresenta-se adequada às demandas informacionais dos usuários estudados, mas a coleção de livros estrangeiros não.
\end{abstract}

Palavras-chave: Biblioteca universitária. Desenvolvimento de coleções. Análise de citações. 


\section{INTRODUÇÃO}

As bibliotecas universitárias surgiram na Idade Média, ligadas a ordens religiosas que deram sustentação ao movimento de criação das universidades. Naquela época, os livros eram manuscritos, o que dificultava e encarecia sua reprodução, apesar disso, as universidades sempre dispuseram de bibliotecas, mesmo que de forma rudimentar (CARVALHO, 2004; VERGER, 1999). Com o passar do tempo, o número de estudantes universitários começa a aumentar, ocasionando um crescimento da produção intelectual. Esse período coincidiu com a decadência da Idade Média, o surgimento do Renascimento e a difusão, na Europa, da tecnologia de reprodução por tipos móveis.

Naturalmente, os reflexos das mudanças trazidas pelo Renascimento atingiram a biblioteca universitária: "quanto mais se lia, mais se produzia conhecimento, o que aumentava o campo para novos estudos. Este ciclo cresceu aumentando a relação entre a universidade, a biblioteca e os seus leitores" (MORIGI; SOUTO, 2005, p. 191). As bibliotecas universitárias se mostraram flexíveis e adaptaram-se às mudanças sociais, resultantes tanto da Revolução Francesa quanto da Revolução Industrial.

No Brasil, as escolas superiores e as bibliotecas acadêmicas surgem entre o final do século XIX e início do século XX, e se encontram, na atualidade, em plena adaptação às mudanças "[...] vivenciadas no final do século XX e início do XXI, que assiste, aceleradamente, à passagem da Sociedade Industrial para a Sociedade da Informação" (CARVALHO, 2004, p. $81)$.

As coleções, que no passado eram formadas de maneira elementar exigem, na atualidade, uma gestão criteriosa e atenta em seu processo de desenvolvimento, de modo que seja possível determinar diretrizes para nortear sua implementação. Deste modo, uma das funções que mais necessita de atenção na administração de uma biblioteca é o desenvolvimento de coleções, um processo que permite identificar os pontos fracos e fortes da coleção, de acordo com as demandas informacionais da sua comunidade usuária (NEGRETE GUTIÉRREZ, 1996).

Essa função, conhecida durante muito tempo apenas como seleção, tem sido destacada na literatura como uma das mais importantes atividades intelectuais do bibliotecário, muito embora a mesma literatura reporte à dificuldade de tornar a teoria uma prática sistemática. Para desempenhar esta atribuição, o bibliotecário deve cumprir um conjunto de atividades específicas que envolvem alocação de recursos financeiros, seleção, aquisição, reposição e descarte de material informacional. E para que essas atividades sejam realizadas de forma que 
atendam ao objetivo último do desenvolvimento de coleções, - a satisfação dos usuários - é importante coletar e organizar os dados referentes ao comportamento dos mesmos, para apoiar a tomada de decisão quanto ao desenvolvimento de coleções (KLAES, 1991).

Smith (1981) sugere que a análise das citações oriundas das teses e dissertações pode servir como um estudo do usuário, pois elas oferecem dados que podem gerar informações valiosas quanto àquele comportamento e servirão para apoiar a tomada de decisão no desenvolvimento de coleções nas bibliotecas acadêmicas. Considerando-se pertinente o proposto por Smith (1981), investigou-se o comportamento de uso dos materiais informacionais dos alunos de doutorado do Programa de Pós-Graduação em Administração (PPGA) da Escola de Administração (EA) da Universidade Federal do Rio Grande do Sul (UFRGS), pela análise das citações expressas nas teses por eles elaboradas.

Apresenta-se neste artigo, uma das etapas da investigação, a que busca responder ao seguinte problema de pesquisa: a coleção de livros da Biblioteca Setorial da EA (BSEA) está adequada às demandas informacionais dos usuários alunos de pós-graduação do PPGA?

O foco desta investigação encontra-se no comportamento de uso do material informacional dos alunos de pós-graduação, mais especificamente os doutorandos, pois os alunos de graduação já se encontram contemplados com uma política de desenvolvimento de coleções que vem sendo desenvolvida pelo Sistema de Bibliotecas da UFRGS (SBU) por meio da metodologia BiblioGrad (STREHL; CASTANHO, 2007). Optou-se pelo estudo das citações utilizadas na elaboração das teses porque elas representam estudos originais, constituem-se numa contribuição real para a especialidade e o material informacional utilizado na sua elaboração apresenta o que há de mais atual sobre o assunto abordado.

A realização deste estudo se justifica porque ele permitirá estabelecer diretrizes para apoiar a tomada de decisão quanto ao desenvolvimento da coleção de livros da Biblioteca Setorial da EA (BSEA), além de atingir três dos cinco objetivos expressos em seu documento de Política de Desenvolvimento de Coleções, a saber: (i) permitir o crescimento racional e equilibrado do acervo; (ii) identificar os elementos adequados à formação da coleção; e (iii) estabelecer prioridades de aquisição de material.

Assim, com o objetivo de determinar a adequação ou não da coleção de livros do SBU para os alunos de doutorado do PPGA, intenciona-se verificar a correlação existente entre os livros citados nas teses por eles elaboradas, de 1999 até 2007, e o acervo disponível no catálogo online (Online Public Access Catalogue - OPAC) do SBU, o SABi ${ }^{1}$.

\footnotetext{
${ }^{1}$ Sistema de Automação de Bibliotecas. Disponível em: http://sabix.ufrgs.br/ALEPH?415825923.
} 


\section{BIBLIOTECA SETORIAL DA ESCOLA DE ADMINISTRAÇÃO DA UFRGS}

Pretendendo-se proporcionar elementos para a contextualização histórica da BSEA, cabe situar o leitor quanto ao SBU, numa breve linha de tempo. Em 1959 foi criado na UFRGS o Serviço Central de Informações Bibliográficas (SCIB), em convênio assinado entre a Universidade e o Conselho Nacional de Pesquisas, por meio do Instituto Brasileiro de Bibliografia e Documentação (IBBD). Em 1962, o SCIB foi extinto e criado, no mesmo ano, o Serviço de Bibliografia e Documentação (SBD).

No ano de 1970, foram aprovados o Estatuto e o Regimento Geral da Universidade, que previam a criação de uma Biblioteca Central, criada efetivamente em 1971 como um Órgão Suplementar diretamente vinculado à Reitoria, com atribuições de coordenar e supervisionar de forma sistêmica o conjunto de bibliotecas da Universidade (BECKER; FORTES, 1961; UNIVERSIDADE FEDERAL DO RIO GRANDE DO SUL, [2008]).

$\mathrm{Na}$ atualidade, a Diretora da Biblioteca Central da UFRGS, Viviane Castanho, coordena tecnicamente um conjunto de 32 bibliotecas: além da Central; 29 setoriais acadêmicas (entre elas a BSEA), duas escolares e uma depositária da ONU.

Por atuar de forma sistêmica, cada biblioteca do SBU constrói seu acervo de acordo com o foco do Instituto, Faculdade ou Escola a que está subordinada administrativamente, salientando-se que os diversos acervos estão disponibilizados para consulta e empréstimo para todos os alunos da UFRGS em qualquer biblioteca do SBU, encontrando-se disponível em um único catálogo eletrônico disponível na internet, o SABi.

Cabe destacar que a coordenação técnica do SBU recomenda que se evite a duplicação do acervo e a compra de material informacional que contenha assunto não pertinente ao assunto de cada biblioteca do Sistema. Por estes motivos e devido à natureza interdisciplinar da Ciência Administrativa, não raro nossos alunos se valem do acervo das diversas bibliotecas do SBU para complementar seus estudos em outras disciplinas. Em outras palavras, o acervo da BSEA não se limita ao material informacional disponível em sua área física, mas abrange o acervo disponível em todas as bibliotecas da UFRGS. 


\section{REFERENCIAL TEÓRICO}

Pode-se afirmar que a Biblioteca de Alexandria, na Antiguidade, representava o resultado da seleção de algumas obras que eram “[...] símbolo da liberdade de expressão e de compromisso com a memória social daquele período". Da mesma forma, na Idade Média, a seleção das obras colecionadas representava o resultado de uma lógica cristã (WEITZEL, 2002, p. 61).

No Renascimento, o processo de seleção foi tratado de modo mais sistemático pelo médico francês Gabriel Naudé (1600-1653), em sua obra Avis pour dresser une bibliothèque e, na Idade Moderna, colecionava-se praticamente tudo o que existia, pois a produção editorial encontrava-se em seu estágio inicial. Comparadas aos padrões atuais, as coleções existentes nas bibliotecas antes do advento da imprensa no ocidente, caracterizavam-se por serem de pequeno porte.

A crescente complexidade das atividades de geração e comunicação da informação provocou uma especialização das bibliotecas e os novos desafios na organização dos estoques de informação distanciaram as unidades de informação entre si. Apesar disso, pontos em comum continuaram existindo, pois, independentemente dos usuários atendidos e das características ambientais, as funções de seleção, aquisição, tratamento, organização, armazenamento e atendimento de referência são encontradas nos mais diversos tipos de bibliotecas (DIAS, 2006).

Contudo, é na segunda metade do século XX que a atividade de desenvolvimento de coleções surge como resposta à explosão bibliográfica devido à irracionalidade de adquirir todo o material informacional que era produzido (FONSECA, 2007; WEITZEL, 2002). Onde surgiu? Nas bibliotecas universitárias de países anglo-saxões em virtude das restrições orçamentárias e da limitação do espaço físico, onde os responsáveis pelo desenvolvimento de coleções precisavam justificar a necessidade de aquisição e racionalizar os recursos disponíveis para o investimento (NEGRETE GUTIÉRREZ, 1993).

Os fenômenos "especialização das bibliotecas" e "explosão bibliográfica" remetem-nos aos questionamentos de Vergueiro (1993, p. 19): “Afinal, estão as coleções sendo realmente desenvolvidas com critérios neste país? Estão elas seguindo qualquer tipo de parâmetro para seu desenvolvimento?"

A preocupação com a política de desenvolvimento de coleções na biblioteca universitária brasileira surge entre o final da década de 70 e início de 80 do século passado. Na ocasião, Antonio Miranda enumerava os problemas enfrentados para formar coleções nestas 
bibliotecas, tais como censura e inflação; e Nice Figueiredo pesquisava, por solicitação da Coordenação de Aperfeiçoamento de Pessoal de Nível Superior (Capes), a situação da seleção e aquisição de material bibliográfico nas bibliotecas acadêmicas. Naqueles tempos não havia literatura em português sobre $\mathrm{o}$ assunto e o ensino da disciplina de Formação e Desenvolvimento de Coleções tinha sido recém introduzido nas escolas de Biblioteconomia (WEITZEL, 2006).

Williams e Fletcher (2006) asseveram que no contexto das bibliotecas universitárias o desenvolvimento de coleções requer a compreensão das demandas informacionais dos autores das teses. Estas demandas podem ser difíceis de discernir, mas a análise de citações possibilitará a compreensão clara de sua natureza.

$\mathrm{Na}$ literatura, são encontrados alguns autores que consideram possível estudar a comunidade usuária pela análise das citações oriundas das teses e dissertações, pois esta análise pode servir como método indireto de estudo do usuário (SMITH, 1981; TARAPANOFF; MIRANDA; ARAÚJO JÚNIOR, 2004; TUÑÓN; BRYDGES, 2005; URBANO SALIDO, 2001), dado que este tipo de investigação permite desenvolver estruturas teóricas a respeito da expectativa do uso dos materiais futuros, baseados nos critérios comuns dos materiais já utilizados (KOVACS, 1990).

Concorda-se com os autores que consideram a análise de citações um estudo da comunidade usuária como um método indireto de análise de comportamento do leitor no uso da informação. Acredita-se que sejam particularmente atraentes, pois as respostas obtidas a partir da análise das citações utilizadas na elaboração das teses apresentam a vantagem de não ser contaminadas por opiniões dos usuários participantes da investigação. Além disso, as informações podem ser descritas e contadas e os dados podem ser coletados com relativa facilidade (TUÑÓN; BRYDGES, 2005).

Pretende-se instrumentalizar o bibliotecário para o planejamento do desenvolvimento da coleção de livros da BSEA, por meio da investigação do uso da informação técnica e científica pela análise das citações expressas nas teses emanadas do PPGA, funcionando como um mecanismo que permite adequar a coleção às demandas informacionais de seus usuários.

\section{MATERIAL E MÉTODO}

Algumas metodologias tentam identificar a relevância das coleções existentes estudando a 
circulação e o uso dos materiais disponíveis na biblioteca, entre elas se destaca a Bibliometria, mais especificamente a análise de citações, que se aplica perfeitamente às coleções de bibliotecas universitárias ou especializadas.

Encontram-se na literatura várias definições para Bibliometria, tais como a elaborada por Foresti (1989, p. 7), considerando-a “[...] uma área extensa da Ciência da Informação que abrange todos os estudos que procuram quantificar os processos de comunicação escrita, aplicando métodos numéricos específicos”. Ou a elaborada por Reitz (2007, tradução nossa), que define Bibliometria como "[...] utilização de métodos matemáticos e estatísticos para estudar e identificar padrões de uso de materiais e serviços dentro de uma biblioteca, ou para analisar a evolução histórica de um corpo específico da literatura, especialmente sua autoria, publicação e utilização".

A análise de citações consiste na contagem da frequência das referências bibliográficas contidas nos documentos. Pode ser feita a partir de uma ordenação linear, ou, na relação entre artigos científicos que geram e recebem citação numa ordenação multidimensional, possibilitando, neste caso, a análise de acoplamento bibliográfico desenvolvida por Kessler em 1963. Ambas as ordenações objetivam determinar padrões acadêmicos de comunicação. Neste estudo utilizaremos a contagem de frequência das referências bibliográficas a partir de uma ordenação linear.

Citações e referências são elementos básicos para os estudos bibliométricos e muitas vezes estes termos são usados de forma indiscriminada para indicar a localização ou identificação de um item informacional. Entretanto, citações e referências são elementos com personalidade própria, que apresentam importante ligação entre si, representada pela indispensável indicação da fonte de onde foi extraída a citação, por meio de uma lista ordenada dos documentos efetivamente citados no texto.

A citação é um excerto, uma menção ao conteúdo de um item informacional que pode ser transcrito literalmente do documento original, a chamada citação direta; ou transcrito de forma interpretada, fazendo uma alusão ao conteúdo do documento, a chamada citação indireta. "As citações bibliográficas textuais servem para dar maior clareza e autoridade ao texto, relacionando as ideias expostas com ideias defendidas em outros trabalhos por outros autores” (ASSOCIAÇÃO BRASILEIRA DE NORMAS TÉCNICAS, 1989, p. 6).

Já a referência é "um conjunto padronizado de elementos descritivos, retirados de um documento que permite sua identificação individual" (ASSOCIAÇÃO BRASILEIRA DE NORMAS TÉCNICAS, 2002, p. 2), viabilizando a representação do documento que foi citado de forma a tornar possível sua localização para consulta na massa documental 
disponível.

Após as breves conceituações acerca do método, iniciou-se a definição do objeto de estudo. Selecionou-se os dados empíricos através da consulta ao $\mathrm{SABi}$, que mantém os dados catalográficos de todas as teses defendidas na UFRGS. Os critérios definidos para a consulta ao catálogo estabelecem que, para pertencer ao universo da pesquisa, a tese cujas citações serão investigadas deve ter duas características: (a) ter sido defendida no PPGA de 1999 até 2007; e (b) ter sido orientada por professore pertencente ao Departamento de Ciências Administrativas (DCA).

Destaca-se que 1999 foi o ano em que ocorreram as primeiras defesas de tese do PPGA, já que o curso de doutorado teve início em 1994. Atualmente, o curso encontra-se estruturado em seis áreas de concentração: Contabilidade e Finanças; Gestão da Tecnologia e da Produção; Marketing; Organizações; Recursos Humanos e Sistemas de Informação e de Apoio à Decisão.

Para proceder à consulta, selecionou-se no $\mathrm{SABi}$, no ícone catálogo, o material do tipo teses e dissertações. Logo após, inseriu-se, no campo de pesquisa, a seguinte linguagem de comando - expressão de busca - por meio da pesquisa Commom Comand Language (CCL):

\section{WUN=adm01 and WPI=pd and WYR=1999 $->2007$}

Ou seja,WUN para produção intelectual do DCA (adm01), WPI para selecionar somente as teses (pd), WYR para delimitar o recorte temporal a ser estudado (1999 -> 2007).

Levando-se em consideração o tempo necessário para realizar a inserção dos dados no banco de dados da investigação, bem como as etapas posteriores de tratamento e interpretação dos mesmos, a consulta ao catálogo foi realizada em abril de 2008.

Foram recuperadas 95 teses e, entre elas, dez não obedeciam aos critérios estabelecidos para esta pesquisa, tendo sido descartadas. Oito foram orientadas por professores do DCA, mas os alunos pertenciam a outro programa de pós-graduação da UFRGS e de duas, os professores pertencentes ao DCA são autores, restando assim 85 teses apresentadas na Tabela 1. 


\begin{tabular}{|c|c|c|c|c|c|c|c|c|c|c|c|}
\hline \multirow{2}{*}{$\begin{array}{l}\text { ÁREA } \\
\text { CONCENTRAÇÃO }\end{array}$} & \multicolumn{9}{|c|}{ ANO } & \multirow{2}{*}{ TOTAL } & \multirow{2}{*}{$(\%)$} \\
\hline & 1999 & 2000 & 2001 & 2002 & 2003 & 2004 & 2005 & 2006 & 2007 & & \\
\hline GTP & 1 & 1 & 2 & 2 & 0 & 4 & 6 & 7 & 0 & 23 & 27,06 \\
\hline MKT & 1 & 1 & 3 & 1 & 1 & 2 & 4 & 3 & 2 & 18 & 21,18 \\
\hline SIAD & 2 & 3 & 2 & 0 & 0 & 2 & 3 & 4 & 0 & 16 & 18,82 \\
\hline ORG & 0 & 1 & 1 & 1 & 2 & 2 & 3 & 3 & 1 & 14 & 16,47 \\
\hline RH & 0 & 2 & 4 & 0 & 0 & 1 & 2 & 0 & 2 & 11 & 12,94 \\
\hline $\mathrm{CF}$ & 0 & 0 & 0 & 0 & 0 & 0 & 2 & 1 & 0 & 3 & 3,53 \\
\hline TOTAL & 4 & 8 & 12 & 4 & 3 & 11 & 20 & 18 & 5 & 85 & 100 \\
\hline$(\%)$ & 4,71 & 9,41 & 14,12 & 4,71 & 3,53 & 12,94 & 23,53 & 21,18 & 5,88 & 100 & \\
\hline
\end{tabular}

Tabela 1: Teses defendidas no PPGA/EA/UFRGS por ano e por área de concentração

Fonte: Elaborado com informações obtidas junto ao SABi (abril, 2008)

Nota: GTP $=$ Gestão da Tecnologia e da Produção, $M K T=$ Marketing, $\mathrm{ORG}=$ Organizações, $R H=$ Recursos Humanos, SIAD = Sistemas de Informação e de Apoio à Decisão e CF = Contabilidade e Finanças

Reunidas as informações sobre cada uma das 85 teses, tais como número do sistema no $\mathrm{SABi}$; ano de defesa; área de concentração; frequência de citações; nome do autor; nome do orientador e título, procedeu-se à contabilização das citações para, em seguida, produzir informações mais refinadas. Foram tabuladas 13.298 citações utilizadas na elaboração das 85 teses, cuja distribuição por área de concentração está sumariada na Tabela 2.

\begin{tabular}{lrrrr}
\hline \multirow{2}{*}{ ÁREA CONCENTRAÇÃO } & \multicolumn{2}{c}{ TESES } & \multicolumn{3}{c}{ CITAÇÕES } \\
\cline { 2 - 5 } & FREQ. & $\mathbf{( \% )}$ & \multicolumn{1}{c}{ FREQ. } & $\mathbf{( \% )}$ \\
\hline GTP & 23 & 27,06 & 3568 & 26,83 \\
MKT & 18 & 21,18 & 2903 & 21,83 \\
SIAD & 16 & 18,82 & 2108 & 15,85 \\
ORG & 14 & 16,47 & 2276 & 17,12 \\
RH & 11 & 12,94 & 2142 & 16,11 \\
CF & 3 & 3,53 & 301 & 2,26 \\
\hline TOTAL & $\mathbf{8 5}$ & $\mathbf{1 0 0}$ & $\mathbf{1 3 2 9 8}$ & $\mathbf{1 0 0}$ \\
\hline
\end{tabular}

Tabela 2:Citações utilizadas nas teses defendidas no PPGA/EA/UFRGS por área de concentração Fonte: Elaborado pelas autoras

Nota: GTP $=$ Gestão da Tecnologia e da Produção, MKT $=$ Marketing, $O R G=$ Organizações, $R H=$ Recursos Humanos, $\mathrm{SIAD}=$ Sistemas de Informação e de Apoio à Decisão e CF = Contabilidade e Finanças

Para classificar as referências, foi elaborada uma lista de possíveis tipologias, de acordo com as normas para elaboração de referências da Associação Brasileira de Normas Técnicas (2002), que norteiam a descrição das referências citadas nas teses estudadas. Apresentam-se detalhadamente as tipologias encontradas na Tabela 3. 


\begin{tabular}{|c|c|c|c|}
\hline \multirow{2}{*}{ NUM. } & \multirow{2}{*}{ TIPOLOGIA } & \multicolumn{2}{|c|}{ FREQUÊNCIA } \\
\hline & & SIMPLES & $(\%)$ \\
\hline 1 & Parte de publicação periódica & 4.984 & 37,47 \\
\hline 2 & Livro & 4.693 & 35,28 \\
\hline 3 & Capítulo de livro & 1.445 & 10,86 \\
\hline 4 & Trabalho apresentado em evento & 614 & 4,61 \\
\hline 5 & Site & 467 & 3,51 \\
\hline 6 & Trabalho apresentado em evento em meio eletrônico & 189 & 1,42 \\
\hline 7 & Tese & 168 & 1,26 \\
\hline 8 & Dissertação & 155 & 1,17 \\
\hline 9 & Não definido & 130 & 0,98 \\
\hline 10 & Livro em meio eletrônico & 106 & 0,80 \\
\hline 11 & Artigo e/ou matéria de jornal & 89 & 0,67 \\
\hline 12 & Parte de publicação periódica em meio eletrônico & 82 & 0,62 \\
\hline 13 & Documento jurídico & 36 & 0,27 \\
\hline 14 & Apud & 29 & 0,22 \\
\hline 15 & Relatório de pesquisa & 28 & 0,21 \\
\hline 16 & Documento jurídico em meio eletrônico & 21 & 0,16 \\
\hline 17 & Capítulo de livro em meio eletrônico & 15 & 0,11 \\
\hline 18 & Evento como um todo & 15 & 0,11 \\
\hline 19 & Bases de dados & 8 & 0,06 \\
\hline 20 & Norma técnica & 5 & 0,04 \\
\hline 21 & TCC Graduação & 5 & 0,04 \\
\hline 22 & Mensagens eletrônicas & 4 & 0,03 \\
\hline 23 & Publicação periódica como um todo & 2 & 0,02 \\
\hline 24 & TCC Especialização & 2 & 0,02 \\
\hline 25 & Lista de discussão & 2 & 0,02 \\
\hline 26 & Evento como um todo em meio eletrônico & 1 & 0,01 \\
\hline 27 & Tese em meio eletrônico & 1 & 0,01 \\
\hline 28 & Dissertação em meio eletrônico & 1 & 0,01 \\
\hline 29 & Artigo e/ou matéria de jornal em meio eletrônico & 1 & 0,01 \\
\hline TOTAL & & 13.298 & 100 \\
\hline
\end{tabular}

Tabela 3: Tipologia dos documentos citados na elaboração das teses defendidas de 1999 até 2007

Fonte: Elaborado pelas autoras

Tomaremos como parâmetro para a análise dos dados o estudo empreendido por Leiding (2005), que examinou um recorte temporal de dez anos das dissertações da biblioteca da James Madison University (JMU), visando determinar a adequação da coleção da biblioteca para a pesquisa de aluno de pós-graduação.

Leiding (2005) definiu como objeto de análise 674 dissertações defendidas de 1993 até 2002. O autor utilizou a técnica da amostra casual simples, por meio da importação dos números de chamada das dissertações para o software Excel onde definiu uma amostra de 101 dissertações (15\%), utilizando o gerador de números aleatórios do programa.

Os dados foram analisados por tipo de material, ano e disciplina, e as citações foram verificadas no catálogo online para determinar a disponibilidade do material informacional na biblioteca. As taxas de disponibilidade local foram calculadas e analisadas para avaliar como as coleções locais servem aos usuários pesquisados. Em seu estudo, Leiding (2005) encontrou os seguintes índices para a disciplina de Administração: os livros foram os materiais 
informacionais mais utilizados na elaboração das teses, representando 48,4\% da amostra.

De acordo com a tabulação dos dados apresentados na Tabela 3, somando-se os livros e capítulos de livros, estes foram os materiais informacionais mais utilizados na elaboração das teses do PPGA, representando 47,05\% da amostra, valor bastante próximo do encontrado por Leiding (2005) em sua investigação, esta similaridade nos permite comparar os dois estudos. Para realizar a análise de interesse desta investigação, destacam-se os livros e capítulos de livros, independentemente de seu suporte físico e por ano de publicação, na Tabela 4.

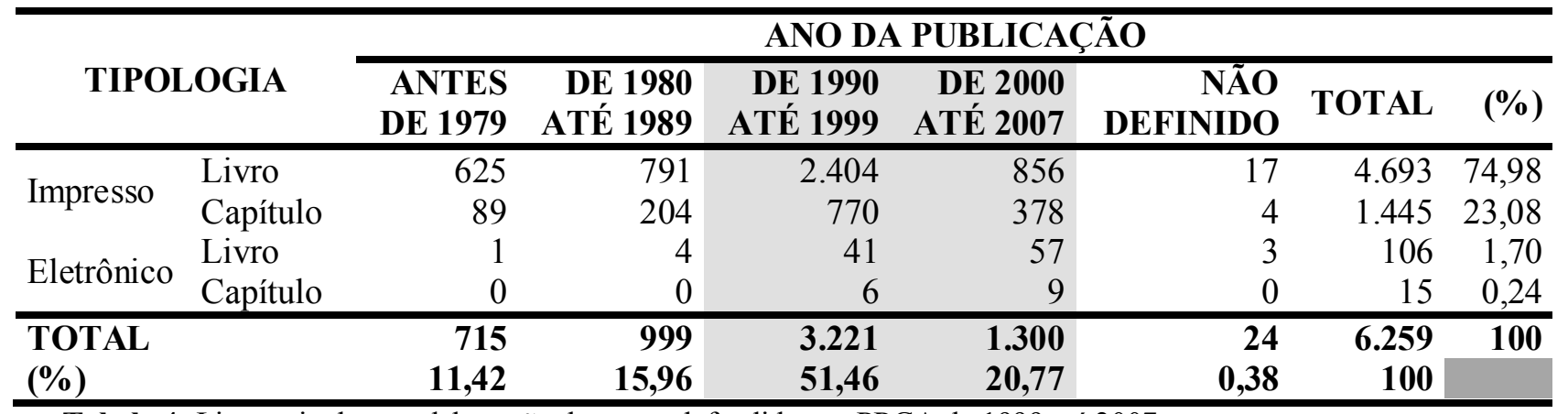

Tabela 4: Livros citados na elaboração das teses defendidas no PPGA de 1999 até 2007

Fonte: Elaborado pelas autoras

Visto que o objetivo da investigação é determinar a adequação ou não da coleção por meio da correlação existente entre os livros citados nas teses estudadas e o acervo disponível no SBU, descartou-se os livros e capítulos de livros em meio eletrônico disponíveis na web, já que os mesmos não se encontram catalogados no SABi.

Devido à maior concentração dos livros citados apresentarem-se de 1990 até 2007, representando $72,23 \%$ das citações coletadas para este tipo de material, definiu-se que este período seria o recorte temporal ideal para a pesquisa. Determinados os parâmetros, apresenta-se na Tabela 5 a amostra definida para esta investigação.

\begin{tabular}{lrrrrr}
\hline \multirow{2}{*}{ TIPOLOGIA } & \multicolumn{5}{c}{ ANO DA PUBLICAÇÃO } \\
\cline { 2 - 6 } & DE 1990 ATÉ 1999 & DE 2000 ATÉ 2007 & TOTAL & $\mathbf{( \% )}$ \\
\hline Livro & 2.404 & 856 & 3260 & 73,95 \\
Capítulo & 770 & 378 & 1.148 & 26,05 \\
\hline TOTAL & $\mathbf{3 . 1 7 4}$ & $\mathbf{1 . 2 3 4}$ & $\mathbf{4 . 4 0 8}$ & $\mathbf{1 0 0}$ \\
$(\%)$ & $\mathbf{7 2}$ & $\mathbf{2 8}$ & $\mathbf{1 0 0}$ & \\
\hline
\end{tabular}

Tabela 5: Amostra

Fonte: Elaborado pelas autoras

Definida a amostra, procedeu-se a exportação das 4.408 referências para o software Excel para o exame das citações de livros e capítulos de livros publicados de 1990 até 2007 . No 
arquivo em Excel, além do campo referência, que continha os elementos descritivos retirados dos livros citados e que permitiam sua identificação individual, foram criados os campos: (a) frequência, para determinar a quantidade de referências (livros) idênticas; (b) idioma, para determinar se a publicação é nacional ou estrangeira; (c) década, para determinar se o livro foi publicado na década de 1990 ou de 2000 até 2007; e (d) SABi, para verificar a existência, ou não, da correlação citação/existência no catálogo.

Além disso, com o exame das referências, visou-se descartar outros tipos de materiais que porventura tenham sido classificados erroneamente como livro, por má elaboração da respectiva referência, por parte dos autores das teses.

Entre as referências elaboradas de forma equivocada, encontraram-se relatórios de pesquisa, textos para discussão, working papers, dissertações, artigos de periódico e anais de eventos que tiveram suas referências elaboradas como se livros fossem. Os equívocos representaram 334 citações, ou 7,57\% do total de citações classificadas erroneamente como livro. Depurada a amostra, iniciou-se a pesquisa no SABi para verificar a existência ou não, no acervo do SBU, dos livros citados pelos autores das 85 teses estudadas, um a um. Os resultados da busca serão apresentados na próxima seção.

\section{ANÁLISE}

Feita a depuração na amostra, restaram 4.074 citações distribuídas em 2.433 livros efetivamente incluídos na pesquisa e sumariados na Tabela 6 .

\begin{tabular}{llrrrr}
\hline LIVROS & ANO DA PUBLICAÇÃO & FREQUENCIA & $\mathbf{( \% )}$ & CITAÇÃO & $\mathbf{( \% )}$ \\
\hline \multirow{2}{*}{ Nacionais } & Década de 1990 & 863 & 35,47 & 1472 & 36,13 \\
& De 2000 até 2007 & 493 & 20,26 & 897 & 22,02 \\
\hline \multirow{2}{*}{ Subtotal } & & $\mathbf{1 . 3 5 6}$ & $\mathbf{5 5 , 7 3}$ & $\mathbf{2 3 6 9}$ & $\mathbf{5 8 , 1 5}$ \\
\multirow{2}{*}{ Estrangeiros } & Década de 1990 & 858 & 35,27 & 1381 & 33,90 \\
& De 2000 até 2007 & 219 & 9,00 & 324 & 7,95 \\
\hline \multirow{2}{*}{ Subtotal } & & $\mathbf{1 . 0 7 7}$ & $\mathbf{4 4 , 2 7}$ & $\mathbf{1 . 7 0 5}$ & $\mathbf{4 1 , 8 5}$ \\
\hline TOTAL & & $\mathbf{2 . 4 3 3}$ & $\mathbf{1 0 0}$ & $\mathbf{4 . 0 7 4}$ & $\mathbf{1 0 0}$ \\
\hline
\end{tabular}

Tabela 6: Livros incluídos na pesquisa

Fonte: Elaborado pelas autoras

Nota-se na Tabela 6, que a razão citação/frequência é igual a 1,74 para os livros nacionais (2.369/1.356); e 1,58 para os livros estrangeiros (1.705/1.077), cada livro citado menos de 
duas vezes. No estudo de Leiding (2005), esta razão, para a disciplina de Administração, é igual a 1,38 (169/122), menor que os resultados obtidos para o uso do acervo do SBU, identificando-se outra similaridade com o estudo escolhido como parâmetro.

Agrupados os 2.433 livros citados por ano de publicação, verificou-se sua existência no acervo do SBU, apresentando-se os resultados na Tabela 7.

\begin{tabular}{lrrrrr}
\hline \multirow{2}{*}{ ANO DA PUBLICAÇÃO } & \multirow{2}{*}{ FREQUÊNCIA } & \multicolumn{4}{c}{ TEM NO SABi? } \\
\cline { 3 - 6 } & SIM & $\mathbf{( \% )}$ & NÃO & $\mathbf{( \% )}$ \\
\hline Década de 1990 & 1721 & 762 & 44,28 & 959 & 55,72 \\
De 2000 até 2007 & 712 & 335 & 47,05 & 377 & 52,95 \\
\hline TOTAL & $\mathbf{2 . 4 3 3}$ & $\mathbf{1 . 0 9 7}$ & & $\mathbf{1 . 3 3 6}$ & \\
\hline
\end{tabular}

Tabela 7: Total de livros citados

Fonte: Elaborado pelas autoras

Analisaram-se a amostra desconsiderando sua procedência, se nacionais ou estrangeiros, verificou-se sua presença no SABi, e, portanto no acervo do SBU. O resultado tanto para os livros publicados na década de 1990, quanto para os publicados de 2000 até 2007, fica abaixo de 50\% no período estudado. Os resultados do estudo de Leiding (2005) para a disciplina de Administração apresentou 72,2\% dos livros citados como existentes no acervo. Apesar de o índice de citação desta pesquisa estar abaixo da pesquisa americana, percebe-se um aumento na existência de livros publicados de 2000 até 2007 no acervo do SBU, em relação aos livros publicados na década de 1990 (2,77\%).

As diferenças percentuais, apesar de apontarem para uma baixa correlação existência do livro no acervo do SBU e seu uso na elaboração das teses do PPGA, indicam que os dados merecem uma abordagem mais verticalizada. Primeiramente, para melhorar e refinar a análise dividiu-se a amostra em dois grupos de livros citados: nacionais e estrangeiros. Além disso, tabularam-se separadamente os dados dos dois grupos de acordo com as citações recebidas e sua existência ou não no catálogo para verificar a proporção dos livros mais citados que fazem parte do acervo.

A Tabela 8 apresenta a correlação entre os livros nacionais citados nas 85 teses e a existência dos mesmos no acervo do SBU. 


\begin{tabular}{|c|c|c|c|c|c|}
\hline \multirow{2}{*}{ ANO DA PUBLICAÇÃO } & \multirow{2}{*}{ FREQUENCIA } & \multicolumn{4}{|c|}{ TEM NO SABi? } \\
\hline & & SIM & $(\%)$ & NÃO & $(\%)$ \\
\hline Década de 1990 & 863 & 599 & 69,41 & 264 & 30,59 \\
\hline De 2000 até 2007 & 493 & 296 & 60,04 & 197 & 39,96 \\
\hline TOTAL & 1.356 & 895 & & 461 & \\
\hline
\end{tabular}

Tabela 8: Livros nacionais

Fonte: Elaborado pelas autoras

Os livros nacionais publicados de 2000 até 2007 encontram-se significativamente presentes no acervo do SBU (60,04\%), apesar do considerável decréscimo em relação aos livros nacionais publicados na década de 1990 (69,41\%), utilizados na elaboração das teses estudadas. Quando se observa os dados da investigação por esta perspectiva, os dados apresentam indícios que o acervo de livros nacionais do SBU revela-se próximo do adequado aos usuários alunos do PPGA, se comparados aos resultados da pesquisa de Leiding (2005).

Encontra-se outro indício desta adequação, como demonstrado na Tabela 9, no fato de doze dos livros mais citados (de 28 até 10 vezes), pertencentes à década de 1990, encontrarem-se na coleção do SBU. Além disso, quando se examina a amostra sob a perspectiva das citações recebidas pelos livros nacionais da década de 1990 existentes no acervo do SBU (78,26\%), o uso destes na elaboração das teses mostrou-se significativo.

\begin{tabular}{|c|c|c|c|c|c|c|c|c|c|c|c|c|c|c|c|c|}
\hline \multirow{2}{*}{\multicolumn{2}{|c|}{$\begin{array}{r}\text { FREQ. } \\
\text { LIVROS } \\
\text { (A) }\end{array}$}} & \multirow[b]{2}{*}{$(\%)$} & \multirow[b]{2}{*}{$\begin{array}{r}\text { FREQ. } \\
\text { CITAÇÕES } \\
\text { (B) }\end{array}$} & \multirow[b]{2}{*}{$\begin{array}{r}\text { (A) } \\
\mathbf{x} \\
(\mathbf{B})\end{array}$} & \multirow[b]{2}{*}{ ACUM. } & \multirow[b]{2}{*}{$(\%)$} & \multicolumn{10}{|c|}{ EXISTE O LIVRO NO ACERVO DO SBU? } \\
\hline & & & & & & & $\begin{array}{r}\text { SIM } \\
\text { (C) }\end{array}$ & $(\%)$ & $\begin{array}{r}\text { (B) } \\
\mathbf{x} \\
(\mathrm{C})\end{array}$ & $\begin{array}{r}\text { CITAÇÕES } \\
\text { ACUM. }\end{array}$ & $(\%)$ & $\begin{array}{r}\text { NÃO } \\
\text { (D) }\end{array}$ & $(\%)$ & $\begin{array}{r}\text { (B) } \\
\mathbf{x} \\
\text { (D) }\end{array}$ & $\begin{array}{r}\text { CITAÇÕES } \\
\text { ACUM. }\end{array}$ & (\%) \\
\hline & 1 & 0,12 & 28 & 28 & 28 & 1,90 & 1 & 0,17 & 28 & 28 & 2,43 & 0 & 0 & 0 & 0 & 0 \\
\hline & 1 & 0,12 & 24 & 24 & 52 & 3,53 & 1 & 0,17 & 24 & 52 & 4,51 & 0 & 0 & 0 & 0 & 0 \\
\hline & 1 & 0,12 & 21 & 21 & 73 & 4,96 & 1 & 0,17 & 21 & 73 & 6,34 & 0 & 0 & 0 & 0 & 0 \\
\hline & 2 & 0,23 & 19 & 38 & 111 & 7,54 & 2 & 0,33 & 38 & 111 & 9,64 & 0 & 0 & 0 & 0 & 0 \\
\hline & 1 & 0,12 & 16 & 16 & 127 & 8,63 & 1 & 0,17 & 16 & 127 & 11,02 & 0 & 0 & 0 & 0 & 0 \\
\hline & 1 & 0,12 & 13 & 13 & 140 & 9,51 & 1 & 0,17 & 13 & 140 & 12,15 & 0 & 0 & 0 & 0 & 0 \\
\hline & 1 & 0,12 & 12 & 12 & 152 & 10,32 & 1 & 0,17 & 12 & 152 & 13,19 & 0 & 0 & 0 & 0 & 0 \\
\hline & 2 & 0,23 & 11 & 22 & 174 & 11,82 & 2 & 0,33 & 22 & 174 & 15,10 & 0 & 0 & 0 & 0 & 0 \\
\hline & 2 & 0,23 & 10 & 20 & 194 & 13,18 & 2 & 0,33 & 20 & 194 & 16,84 & 0 & 0 & 0 & 0 & 0 \\
\hline & 4 & 0,46 & 9 & 36 & 230 & 15,62 & 3 & 0,50 & 27 & 221 & 19,18 & 1 & 0,38 & 9 & 9 & 2,81 \\
\hline & 2 & 0,23 & 8 & 16 & 246 & 16,71 & 2 & 0,33 & 16 & 237 & 20,57 & 0 & 0 & 0 & 9 & 2,81 \\
\hline & 6 & 0,70 & 7 & 42 & 288 & 19,56 & 5 & 0,83 & 35 & 272 & 23,61 & 1 & 0,38 & 7 & 16 & 5,00 \\
\hline & 5 & 0,58 & 6 & 30 & 318 & 21,60 & 5 & 0,83 & 30 & 302 & 26,22 & 0 & 0,00 & 0 & 16 & 5,00 \\
\hline & 17 & 1,97 & 5 & 85 & 403 & 27,38 & 16 & 2,67 & 80 & 382 & 33,16 & 1 & 0,38 & 5 & 21 & 6,56 \\
\hline & 19 & 2,20 & 4 & 76 & 479 & 32,54 & 19 & 3,17 & 76 & 458 & 39,76 & 0 & 0,00 & 0 & 21 & 6,56 \\
\hline & 36 & 4,17 & 3 & 108 & 587 & 39,88 & 30 & 5,01 & 90 & 548 & 47,57 & 6 & 2,27 & 18 & 39 & 12,19 \\
\hline & 123 & 14,25 & 2 & 246 & 833 & 56,59 & 97 & 16,19 & 194 & 742 & 64,41 & 26 & 9,85 & 52 & 91 & 28,44 \\
\hline & 639 & 74,04 & 1 & 639 & 1472 & 100 & 410 & 68,45 & 410 & 1152 & 100 & 229 & 86,74 & 229 & 320 & 100 \\
\hline TOTAL & 863 & & & 1472 & & & 599 & & & 1152 & & 264 & & & 320 & \\
\hline (\%) & & 100 & & 100 & & & & 100 & & 78,26 & & & 100 & & 21,74 & \\
\hline
\end{tabular}

Tabela 9: Livros nacionais publicados na década de 1990: citações recebidas e existência no acervo

Fonte: Elaborado pelas autoras 
Por outro lado, como se apresenta na Tabela 10, os livros nacionais mais citados (de 25 até 10 vezes), publicados de 2000 até 2007, encontram-se na coleção do SBU, à exceção de um. E as citações recebidas pelos livros deste período presentes na coleção do SBU representam $70,24 \%$, muito próximo do valor encontrado por Leiding (2005).

\begin{tabular}{|c|c|c|c|c|c|c|c|c|c|c|c|c|c|c|c|}
\hline \multirow[b]{2}{*}{$\begin{array}{r}\text { FREQ. } \\
\text { LIVROS } \\
\text { (A) }\end{array}$} & \multirow[b]{2}{*}{$(\%)$} & \multirow[b]{2}{*}{$\begin{array}{r}\text { FREQ. } \\
\text { CITAÇÕES } \\
\text { (B) }\end{array}$} & \multirow[b]{2}{*}{$\begin{array}{r}(\mathrm{A}) \\
\mathbf{x} \\
(\mathrm{B})\end{array}$} & \multirow[b]{2}{*}{ ACUM. } & \multirow[b]{2}{*}{$(\%)$} & \multicolumn{10}{|c|}{ EXISTE O LIVRO NO ACERVO DO SBU? } \\
\hline & & & & & & $\begin{array}{r}\text { SIM } \\
\text { (C) }\end{array}$ & $(\%)$ & $\begin{array}{r}\text { (B) } \\
\mathbf{x} \\
(\mathrm{C})\end{array}$ & $\begin{array}{r}\text { CITAÇÕES } \\
\text { ACUM. }\end{array}$ & $(\%)$ & $\begin{array}{r}\text { NÃO } \\
\text { (D) }\end{array}$ & $(\%)$ & $\begin{array}{r}\text { (B) } \\
\mathbf{x} \\
\text { (D) }\end{array}$ & $\begin{array}{r}\text { CITAÇÕES } \\
\text { ACUM. }\end{array}$ & $(\%)$ \\
\hline 2 & 0,41 & 25 & 50 & 50 & 5,57 & 2 & 0,70 & 50 & 50 & 7,94 & 0 & 0 & 0 & 0 & 0,00 \\
\hline 1 & 0,20 & 24 & 24 & 74 & 8,25 & 1 & 0,35 & 24 & 74 & 11,75 & 0 & 0 & 0 & 0 & 0,00 \\
\hline 4 & 0,81 & 15 & 60 & 134 & 14,94 & 3 & 1,05 & 45 & 119 & 18,89 & 1 & 0,48 & 15 & 15 & 5,62 \\
\hline 2 & 0,41 & 14 & 28 & 162 & 18,06 & 2 & 0,70 & 28 & 147 & 23,33 & 0 & 0 & 0 & 15 & 5,62 \\
\hline 1 & 0,20 & 13 & 13 & 175 & 19,51 & 1 & 0,35 & 13 & 160 & 25,40 & 0 & 0 & 0 & 15 & 5,62 \\
\hline 3 & 0,61 & 10 & 30 & 205 & 22,85 & 3 & 1,05 & 30 & 190 & 30,16 & 0 & 0 & 0 & 15 & 5,62 \\
\hline 3 & 0,61 & 8 & 24 & 229 & 25,53 & 3 & 1,05 & 24 & 214 & 33,97 & 0 & 0 & 0 & 15 & 5,62 \\
\hline 4 & 0,81 & 7 & 28 & 257 & 28,65 & 4 & 1,40 & 28 & 242 & 38,41 & 0 & 0 & 0 & 15 & 5,62 \\
\hline 6 & 1,22 & 6 & 36 & 293 & 32,66 & 6 & 2,11 & 36 & 278 & 44,13 & 0 & 0 & 0 & 15 & 5,62 \\
\hline 7 & 1,42 & 5 & 35 & 328 & 36,57 & 3 & 1,05 & 15 & 293 & 46,51 & 4 & 1,92 & 20 & 35 & 13,11 \\
\hline 27 & 5,48 & 3 & 81 & 409 & 45,60 & 18 & 6,32 & 54 & 347 & 55,08 & 9 & 4,33 & 27 & 62 & 23,22 \\
\hline 55 & 11,16 & 2 & 110 & 519 & 57,86 & 44 & 15,44 & 88 & 435 & 69,05 & 11 & 5,29 & 22 & 84 & 31,46 \\
\hline 378 & 76,67 & 1 & 378 & 897 & 100 & 195 & 68,42 & 195 & 630 & 100 & 183 & 87,98 & 183 & 267 & 100 \\
\hline TOTAL 493 & & & 897 & & & 285 & & & 630 & & 208 & & & 267 & \\
\hline$(\%)$ & 100 & & 100 & & & & 100 & & 70,24 & & & 100 & & 29,76 & \\
\hline
\end{tabular}

Tabela 10: Livros nacionais publicados de 2000 até 2007: citações recebidas e existência no acervo

Fonte: Elaborado pelas autoras

Para verificar a correlação entre os livros estrangeiros citados nas teses e a existência dos mesmos no SABi, tabulou-se os dados como apresentados na Tabela 11. A análise dos dados sobre os livros estrangeiros é preocupante, pois os índices de inexistência no acervo das bibliotecas da UFRGS se mostraram altos. Tanto os livros publicados na década de 1990, quanto os livros publicados de 2000 até 2007 tem baixo índice de presença no SABi, ficando abaixo de $20 \%$. No caso específico, pode-se afirmar que o acervo de livros estrangeiros da UFRGS não está atendendo aos doutorandos alunos do PPGA.

\begin{tabular}{lrrrrr}
\hline \multirow{2}{*}{ ANO DA PUBLICAÇÃO } & \multirow{2}{*}{ FREQUENCIA } & \multicolumn{4}{c}{ TEM NO SABi? } \\
\cline { 4 - 7 } & & SIM & $\mathbf{( \% )}$ & NÃO & $\mathbf{( \% )}$ \\
\hline Década de 1990 & 858 & 163 & 19,00 & 695 & 81,00 \\
De 2000 até 2007 & 219 & 39 & 17,81 & 180 & 82,19 \\
\hline TOTAL & $\mathbf{1 . 0 7 7}$ & $\mathbf{2 0 2}$ & & $\mathbf{8 7 5}$ & \\
\hline
\end{tabular}

Tabela 11:Livros estrangeiros

Fonte: Elaborado pelas autoras

Apesar do indício de deficiência, os livros estrangeiros mais citados (de 42 a 10 vezes) na 
elaboração das teses publicados na década de 1990, estão disponíveis no acervo do SBU, com exceção de três (Tabela 12). Este fato poderia sinalizar que esta deficiência na adequação do acervo pode ser decorrência de outros fatores, tais como dificuldades na aquisição e preço, dando-se prioridade para aquisição de livros mais utilizados.

\begin{tabular}{|c|c|c|c|c|c|c|c|c|c|c|c|c|c|c|c|}
\hline \multirow[b]{2}{*}{$\begin{array}{r}\text { FREQ. } \\
\text { LIVROS } \\
(A)\end{array}$} & \multirow[b]{2}{*}{$(\%)$} & \multirow[b]{2}{*}{$\begin{array}{r}\text { FREQ. } \\
\text { CITAÇÕES } \\
\text { (B) }\end{array}$} & \multirow[b]{2}{*}{$\begin{array}{r}(\mathrm{A}) \\
\mathbf{x} \\
(\mathrm{B})\end{array}$} & \multirow[b]{2}{*}{ ACUM. } & \multirow[b]{2}{*}{$(\%)$} & \multicolumn{10}{|c|}{ EXISTE O LIVRO NO ACERVO DO SBU? } \\
\hline & & & & & & $\begin{array}{r}\text { SIM } \\
\text { (C) }\end{array}$ & $(\%)$ & $\begin{array}{r}\text { (B) } \\
\mathbf{x} \\
(\mathrm{C})\end{array}$ & $\begin{array}{r}\text { CITAÇÕES } \\
\text { ACUM. }\end{array}$ & $(\%)$ & $\begin{array}{r}\text { NÃO } \\
\text { (D) }\end{array}$ & $(\%)$ & $\begin{array}{r}\text { (B) } \\
\mathbf{x} \\
\text { (D) }\end{array}$ & $\begin{array}{r}\text { CITAÇÕES } \\
\text { ACUM. }\end{array}$ & $(\%)$ \\
\hline 1 & 0,12 & 42 & 42 & 42 & 3,04 & 0 & 0,00 & 0 & 0 & 0 & 1 & 0,14 & 42 & 42 & 4,25 \\
\hline 1 & 0,12 & 26 & 26 & 68 & 4,92 & 1 & 0,61 & 26 & 26 & 6,63 & 0 & 0,00 & 0 & 42 & 4,25 \\
\hline 1 & 0,12 & 25 & 25 & 93 & 6,73 & 1 & 0,61 & 25 & 51 & 13,01 & 0 & 0,00 & 0 & 42 & 4,25 \\
\hline 1 & 0,12 & 21 & 21 & 114 & 8,25 & 1 & 0,61 & 21 & 72 & 18,37 & 0 & 0,00 & 0 & 42 & 4,25 \\
\hline 1 & 0,12 & 17 & 17 & 131 & 9,49 & 0 & 0,00 & 0 & 72 & 18,37 & 1 & 0,14 & 17 & 59 & 5,97 \\
\hline 1 & 0,12 & 15 & 15 & 146 & 10,57 & 1 & 0,61 & 15 & 87 & 22,19 & 0 & 0,00 & 0 & 59 & 5,97 \\
\hline 1 & 0,12 & 12 & 12 & 158 & 11,44 & 1 & 0,61 & 12 & 99 & 25,26 & 0 & 0,00 & 0 & 59 & 5,97 \\
\hline 1 & 0,12 & 11 & 11 & 169 & 12,24 & 1 & 0,61 & 11 & 110 & 28,06 & 0 & 0,00 & 0 & 59 & 5,97 \\
\hline 2 & 0,23 & 10 & 20 & 189 & 13,69 & 1 & 0,61 & 10 & 120 & 30,61 & 1 & 0,14 & 10 & 69 & 6,98 \\
\hline 3 & 0,35 & 9 & 27 & 216 & 15,64 & 1 & 0,61 & 9 & 129 & 32,91 & 2 & 0,29 & 18 & 87 & 8,80 \\
\hline 1 & 0,12 & 7 & 7 & 231 & 16,73 & 1 & 0,61 & 7 & 136 & 34,69 & 0 & 0,00 & 0 & 95 & 9,61 \\
\hline 3 & 0,35 & 6 & 18 & 249 & 18,03 & 1 & 0,61 & 6 & 142 & 36,22 & 2 & 0,29 & 12 & 107 & 10,82 \\
\hline 15 & 1,75 & 5 & 75 & 324 & 23,46 & 5 & 3,07 & 25 & 167 & 42,60 & 10 & 1,44 & 50 & 157 & 15,87 \\
\hline 19 & 2,21 & 4 & 76 & 400 & 28,96 & 6 & 3,68 & 24 & 191 & 48,72 & 13 & 1,87 & 52 & 209 & 21,13 \\
\hline 34 & 3,96 & 3 & 102 & 502 & 36,35 & 17 & 10,43 & 51 & 242 & 61,73 & 17 & 2,45 & 51 & 260 & 26,29 \\
\hline 107 & 12,47 & 2 & 214 & 716 & 51,85 & 25 & 15,34 & 50 & 292 & 74,49 & 82 & 11,80 & 164 & 424 & 42,87 \\
\hline 665 & 77,51 & 1 & 665 & 1381 & 100,00 & 100 & 61,35 & 100 & 392 & 100 & 565 & 81,29 & 565 & 989 & 100 \\
\hline TOTAL 858 & & & 1381 & & & 163 & & & 392 & & 695 & & & 989 & \\
\hline$(\%)$ & 100 & & 100 & & & & 100 & & 28,38 & & & 100 & & 71,62 & \\
\hline
\end{tabular}

Tabela 12: Livros estrangeiros publicados na década de 1990: citações recebidas e existência no acervo Fonte: Elaborado pelas autoras

Contudo, para os livros estrangeiros mais citados (11 e 4 vezes cada um), publicados de 2000 até 2007, a inadequação do acervo é mais grave, pois dos dez livros mais utilizados pelos doutorandos, o acervo do SBU só dispões de quatro, como sumariado na Tabela 13.

\begin{tabular}{|c|c|c|c|c|c|c|c|c|c|c|c|c|c|c|c|}
\hline \multirow[b]{2}{*}{$\begin{array}{r}\text { FREQ. } \\
\text { LIVROS } \\
\text { (A) }\end{array}$} & \multirow[b]{2}{*}{$(\%)$} & \multirow[b]{2}{*}{$\begin{array}{r}\text { FREQ. } \\
\text { CITAÇÕES } \\
\text { (B) }\end{array}$} & \multirow[b]{2}{*}{$\begin{array}{r}(\mathrm{A}) \\
\mathbf{x} \\
(\mathrm{B})\end{array}$} & \multirow[b]{2}{*}{ ACUM. } & \multirow[b]{2}{*}{$(\%)$} & \multicolumn{10}{|c|}{ EXISTE O LIVRO NO ACERVO DO SBU? } \\
\hline & & & & & & $\begin{array}{r}\text { SIM } \\
\text { (C) }\end{array}$ & $(\%)$ & $\begin{array}{r}\text { (B) } \\
\mathbf{x} \\
(\mathrm{C})\end{array}$ & $\begin{array}{r}\text { CITAÇÕES } \\
\text { ACUM. }\end{array}$ & $(\%)$ & $\begin{array}{r}\text { NÃO } \\
\text { (D) }\end{array}$ & $(\%)$ & $\begin{array}{r}\text { (B) } \\
\mathbf{x} \\
\text { (D) }\end{array}$ & $\begin{array}{r}\text { CITAÇÕES } \\
\text { ACUM. }\end{array}$ & $(\%)$ \\
\hline 8 & 3,65 & 11 & 88 & 88 & 27,16 & 4 & 10,26 & 44 & 44 & 19,73 & 4 & 2,22 & 44 & 44 & 18,26 \\
\hline 2 & 0,91 & 4 & 8 & 96 & 29,63 & 0 & 0,00 & 0 & 44 & 19,73 & 2 & 1,11 & 8 & 52 & 21,58 \\
\hline 19 & 8,68 & 2 & 38 & 134 & 41,36 & 4 & 10,26 & 8 & 52 & 23,32 & 15 & 8,33 & 30 & 82 & 34,02 \\
\hline 190 & 86,76 & 1 & 190 & 324 & 100 & 31 & 79,49 & 31 & 83 & 37,22 & 159 & 88,33 & 159 & 241 & 100 \\
\hline TOTAL 219 & & & 324 & & & 39 & & & 83 & & 180 & & & 241 & \\
\hline$(\%)$ & 100 & & 100 & & & & 100 & & 25,62 & & & 100 & & 74,38 & \\
\hline
\end{tabular}

Tabela 13: Livros estrangeiros publicados de 2000 até 2007: citações recebidas e existência no acervo

Fonte: Elaborado pelas autoras

Dessa forma, tomando como parâmetro o estudo de Leiding (2005), o acervo de livros 
estrangeiros disponíveis no SBU encontra-se inadequado para o uso na elaboração das teses dos doutorandos do PPGA.

\section{CONCLUSÃO}

Concluiu-se com êxito a pesquisa sobre a adequação da coleção de livros do SBU para os usuários alunos de doutorado do PPGA, através da correlação entre os livros citados nas teses estudadas e o acervo disponível no SABi.

De acordo com os resultados obtidos, pode-se responder a pergunta de pesquisa: a coleção de livros nacionais apresenta-se bastante próximo do adequado às demandas informacionais dos usuários, alunos de doutorado do PPGA, enquanto que a coleção de livros estrangeiros não.

Provavelmente a adequação do acervo aos usuários doutorandos do PPGA apresentada nos livros nacionais, deve-se a maior facilidade de aquisição de material bibliográfico nacional, seja por compra, doação ou permuta. Na mesma linha de raciocínio, atribui-se a inadequação do acervo de livros estrangeiros para estes usuários às dificuldades inerentes a compra de material bibliográfico importado em uma universidade pública, por meio de licitação.

De toda forma, como os percentuais de existência dos livros citados no acervo do SBU têm decrescido de 2000 até 2007, independente de ele ser nacional ou estrangeiro, deve-se observar este fenômeno com o devido cuidado, por meio de estudos de usuários mais frequentes que repercutam num desenvolvimento de coleções que atendam as suas demandas informacionais.

Certamente os resultados apresentados nos permitem estabelecer diretrizes para apoiar futuros investimentos em material bibliográfico para o acervo da BSEA, tornando-o assim, mais adequado aos pesquisadores, pois além do diagnóstico obtido sobre o acervo, este estudo produziu uma lista de sugestões que poderão ser utilizadas em futuras aquisições para adequar o acervo à demanda informacional dos usuários pesquisados. 


\section{REFERÊNCIAS}

ASSOCIAÇÃO BRASILEIRA DE NORMAS TÉCNICAS. NBR 6023: informação e documentação - referências - elaboração. Rio de Janeiro, 2002.

NBR 10719: apresentação de relatórios técnico-científicos. Rio de Janeiro, 1989.

CARVALHO, I. C. L. A socialização do conhecimento no espaço das bibliotecas universitárias. Niterói: Intertexto, 2004.

DIAS, E. W. Organização do conhecimento no contexto de bibliotecas tradicionais e digitais. In: NAVES, M. M. L.; KURAMOTO, H. (Org.). Organização da informação: princípios e tendências. Brasília, DF: Briquet de Lemos, 2006. p. 62-75.

FONSECA, E. N. Introdução à Biblioteconomia. 2. ed. Brasília, DF: Briquet de Lemos, 2007.

FORESTI, N. Estudo da contribuição das revistas brasileiras de Biblioteconomia e Ciência da Informação enquanto fonte de referência para a pesquisa. 1989, 209 f.: il. Dissertação (Mestrado) - Departamento de Biblioteconomia, Faculdade de Estudos Sociais e Aplicados, Universidade de Brasília, Brasília, DF, 1989.

KLAES, R. R. Sistema de informação gerencial para desenvolvimento de coleções. Ciência da Informação, Brasília, DF, v. 20, n. 2. p. 220-228, jul./dez. 1991.

KOVACS, B. Decision making in collection development literature. In: . The decision-making process for library collections: case studies in four types of libraries. New York: Greenwood, 1990. p. 12-22.

LEIDING, Reba. Using Citation Checking of Undergraduate Honors Thesis Bibliographies to Evaluate Library Collections. College and Research Libraries, v. 66, n. 5, p. 417-429, September 2005. Disponível em $<$ http://www.ala.org/ala/acrl/acrlpubs/crljournal/backissues2005a/crlsept05/Leiding05.pdf>. Acesso em: 16 jan. 2009.

MORIGI, V. J.; SOUTO, L. R. Entre o passado e o presente: as visões de biblioteca no mundo contemporâneo. Revista ACB: Biblioteconomia em Santa Catarina, v. 10, n. 2, p. 189-206, jan./dez. 2005. 
NEGRETE GUTIÉRREZ, M. C. La comunidad universitaria y el desarrollo de colecciones. In: .; CALVA GONZÁleZ, J. J. (Comp.). Desarrollo de colecciones y diseño de servicios. México, DF: UNAM/Centro Universitário de Investigaciones Bibliotecológicas, 1996. p. 1-4.

El papel del bibliotecólogo en el desarrollo de colecciones en la biblioteca universitaria. Investigación Bibliotecologica: archivonomia, bibliotecologia e información, México, v. 7, n. 14, p. 5-7, ene./jun. 1993.

REITZ, J. M. ODLIS: online dictionary for Library and Information Science. 2007. Disponível em: $<$ http://lu.com/odlis/search.cfm>. Acesso em: 2 out. 2008.

SMITH, L. C. Citation analysis. Library Trends, Illinois, v. 30, n. 1, p. 83-106, Summer 1981.

STREHL, L.; CASTANHO, V. Bibliograd: metodologia de avaliação para o acervo de graduação. 2007. Disponível em: <http://www.biblioteca.ufrgs.br/modulo8-3.ppt>. Acesso em: 07 out. 2008.

TARAPANOFF, K.; MIRANDA, D. M.; ARAÚJO JÚNIOR, R. H. de. (Colab.). Técnicas para tomada de decisão nos sistemas de informação. Brasília, DF: Thesaurus, 2004.

TUNÓN, J.; BRYDGES, B. Improving the quality of university libraries through citation mining and analysis using two new dissertation bibliometric assessment tools. In: WORD LIBRARY AND INFORMATION CONGRESS. IFLA GENERAL CONFERENCE AND COUNCIL, 71. : 2005, Oslo. Libraries: a voyage of discovery. Disponível em: $<$ http://www.ifla.org.sg/IV/ifla71/papers/078e-Tunon_Brydges.pdf $>$. Acesso em: 07 jun. 2006 .

URBANO SALIDO, C. El análisis de citas en trabajos de investigadores como método para el estudio del uso de información en bibliotecas. Anales de Documentación, Murcia, v. 4, p. 243-266, 2001. Disponível em: <http://www.um.es/fccd/anales/ad04/ad0400.html>. Acesso em: 17 jun. 2006.

VERGER, J. Homens e saber na Idade Média. Bauru: Edusc, 1999.

VERGUEIRO, W. C. S. Desenvolvimento de coleções: uma nova visão para o planejamento de recursos informacionais. Ciência da Informação, Brasília, DF, v. 22, n. 1, p. 13-21, jan./abr. 1993. 
WEITZEL, S. R. Elaboração de uma política de desenvolvimento de coleções em bibliotecas universitárias. Rio de Janeiro: Interciência, 2006.

WEITZEL, S. R. O desenvolvimento de coleções e a organização do conhecimento: suas origens e desafios. Perspectivas em Ciência a Informação, Belo Horizonte, v. 7, n. 1, p. 61 67 , jan./jun. 2002

WILLIAMS, V. K.; FLETCHER, C. L. Materials used by master's students in engineering and implications for collection development: a citation analysis. Issues in Science and Technology Librarianship, Chicago, n. 45, winter 2006, Disponível em: $<$ http://www.istl.org/06-winter/refereed1.htm>. Acesso em: 07 jan. 2009.

\begin{abstract}
The aim of this research is to determine the appropriateness of the books collection in the Universidade Federal do Rio Grande do Sul libraries to $\mathrm{PhD}$ students in Management and Business. The method finds correlation between books cited in the PhD thesis from 1999 to 2007, and the book collection available in the libraries. The research concludes that the books collection edited in Portuguese language is according to informational demands of the $\mathrm{PhD}$ students, but the books collection in others languages are not.
\end{abstract}

Keywords: Academic library. Collection development. Citation analisys.

Originais recebidos em: 06/10/2009

Aceito para publicação em: 13/04/2010 